\title{
Distinct Causal Mechanisms of Attentional Guidance by Working Memory and Repetition Priming in Early Visual Cortex
}

\author{
David Soto, ${ }^{1}$ Dafydd Llewelyn, ${ }^{1}$ and Juha Silvanto ${ }^{2,3}$ \\ ${ }^{1}$ Department of Medicine, Centre of Neuroscience, Imperial College London, London SW7 2AZ, United Kingdom, ${ }^{2}$ School of Science, Brain Research Unit, \\ Low Temperature Laboratory, Aalto University, 00076 Aalto, Finland, and ${ }^{3}$ Cognitive Brain Research Unit, Institute of Behavioral Sciences, University of \\ Helsinki, FIN-00014 Helsinki, Finland
}

Human attention may be guided by representations held in working memory (WM) and also by priming from implicit memory. Neurophysiological data suggest that WM and priming may be associated with distinct neural mechanisms, but this prior evidence is only correlative. Furthermore, the role of the visual cortex in attention biases from memory remains unclear, because most previous studies conflated memory and selection processes. Here, we manipulated memory and attention in an orthogonal fashion and used an interventional approach to demonstrate the functional significance of WM and priming states in visual cortex for attentional biasing. Observers searched for a Landolt target that was preceded by a nonpredictive color cue that either had to be held in WM for a later recognition test or merely attended (priming counterpart). The application of transcranial magnetic stimulation (TMS) over the occipital cortex modulated the impact of memory on search. Critically, the direction of this modulation depended on the memory state. In the WM condition, the application of TMS on validly cued trials (when the cue surrounded the sought target) enhanced search accuracy relative to the invalid trials (when the cue surrounded a distracter); the opposite pattern was observed in the priming condition. That the effects of occipital TMS on selection were contingent on memory context demonstrates that WM and priming represent distinct states in the early visual cortex that play a causal role in memory-based guidance of attention.

\section{Introduction}

Current understanding contends that visual selection may be a consequence of feedback from memory signals matching the sought target (Desimone and Duncan, 1995). There are at least two forms of memory effects on selection based on (1) guidance from representations in working memory (WM) (Soto et al., 2007) and (2) implicit priming by stimulus repetition (Kristjánsson and Campana, 2010). WM and priming may be linked to distinct neural mechanisms. Reappearance of a WM item may be associated with elevated activity in temporal and occipital regions (Miller and Desimone, 1994; Motter, 1994; Soto et al., 2007), whereas mere stimulus repetition can attenuate responses in similar regions (Miller and Desimone, 1994; Brown and Xiang, 1998; Soto et al., 2007). However, these neurophysiological data are correlative in nature and therefore silent as to the functional significance of these neural activation patterns for attentional guidance. Notably, most prior studies assessing feature-based attention used cues that specified the target of selection, thus con-

Received Dec. 15, 2011; accepted Jan. 16, 2012.

Author contributions: D.S. and J.S. designed research; D.S. and D.L. performed research; D.S. and J.S. analyzed data; D.S., D.L., and J.S. wrote the paper.

This work was supported by the Medical Research Council of the United Kingdom (Grant 89631) and the Academy of Finland (Grant 137485).

The authors declare no competing financial interests.

Correspondence should be addressed to David Soto at the above address. E-mail: d.soto@imperial.ac.uk.

DOI:10.1523/JNEUROSCI.6243-11.2012

Copyright $\odot 2012$ the authors $\quad 0270-6474 / 12 / 323447-06 \$ 15.00 / 0$ flating neural responses caused by memory and selection (for discussion, see Soto et al., 2007). Even when visual cortical responses may be based on feature cues (Chawla et al., 1999; Martinez-Trujillo and Treue, 2004) they may merely reflect a memory signal for the properties of the target (Pasternak and Greenlee, 2005; Harrison and Tong, 2009). There is no clear evidence to date that visual cortices play a causal role in attentional biasing.

We assayed the role of WM and priming states in visual cortex for attentional guidance by delivering occipital transcranial magnetic stimulation (TMS) as participants performed a memoryguided search task. Observers were presented with color cues to hold in WM or merely attend (priming condition). In valid trials the search target was surrounded by an item matching the color in memory, whereas in invalid trials the memory cue matched a distracter. Memory cues are not predictive of the target but can nevertheless bias attention to matching items (Soto et al., 2007). TMS effects on behavior may depend on the activation state of the stimulated region (Silvanto et al., 2008). We reasoned that if WM and priming are associated with distinct states in visual cortex, which play a causal role in memory guidance of search, then the behavioral impact of occipital TMS could be dissociated across the two memory conditions.

\section{Materials and Methods}

Participants. Thirteen healthy right-handed observers ( 3 males; mean age 22 years) participated in return of monetary compensation. The study 


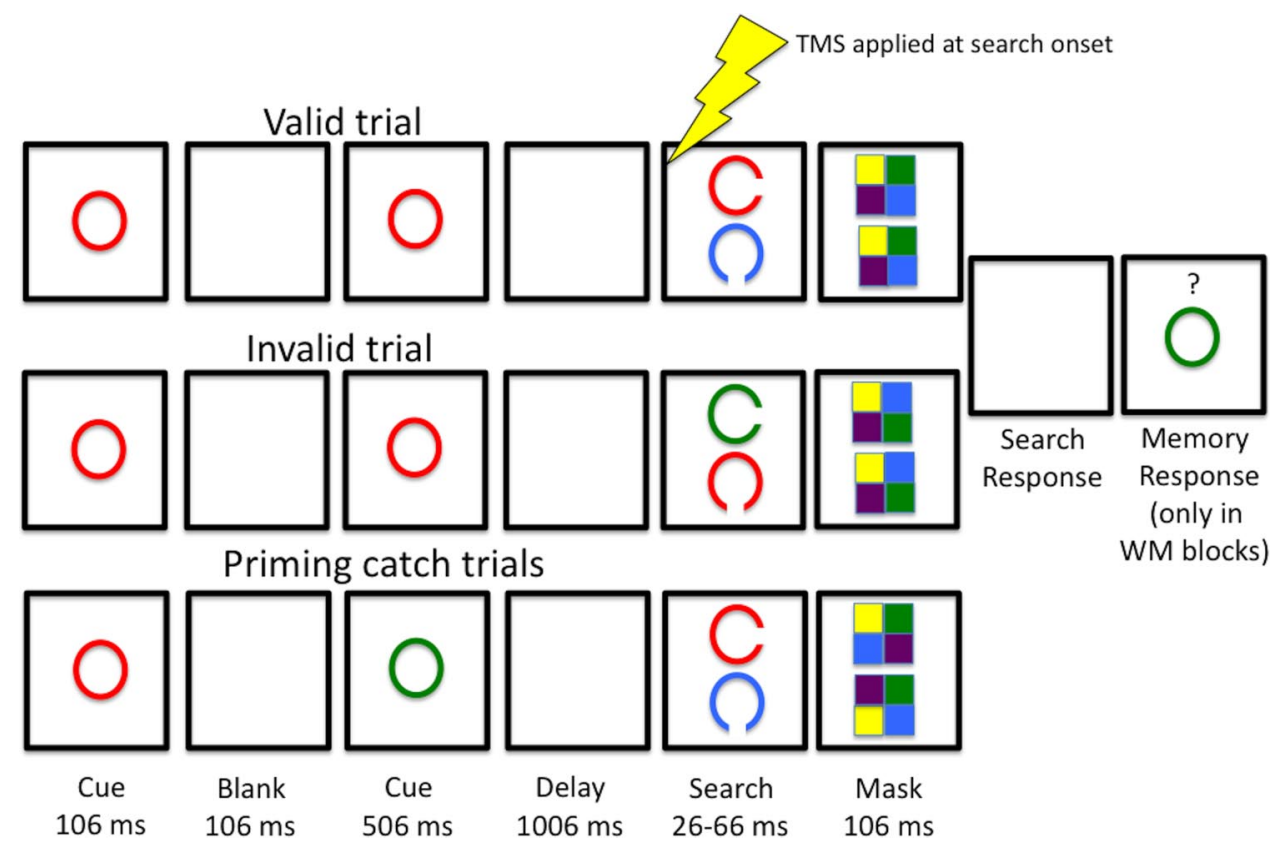

Figure 1. Trial sequences.

was approved by the West London Ethics Committee. One participant was excluded because he did not follow instructions and had chance performance (i.e., 50\% correct) in the search task, thus leaving a sample of 12 participants.

Experimental procedure. Stimuli and task were controlled by E-prime v2.0 (Psychology Software Tools Inc.). Stimuli were viewed binocularly at a distance of $106 \mathrm{~cm}$. Each trial began with an instruction cue ("just look" or "memorize") followed by a blank screen (506 ms) and then by the memory color cue presented at central fixation. The cue could be drawn in five possible colors (red, green, blue, yellow, and pink) and was flashed twice, for $106 \mathrm{~ms}$ and then for $506 \mathrm{~ms}$, with a $106 \mathrm{~ms}$ blank interval. In WM blocks, participants had to hold the cue in WM, whereas in priming blocks they had to merely attend it (Fig. 1). To ensure the cue was attended in the priming condition, $10 \%$ catch trials were included where participants had to report whether the color of the cue changed from the first to second presentation (Fig. 1, bottom). The search display followed $1 \mathrm{~s}$ after cue offset. One-second delay is a suitable timeframe for both WM and priming because priming effects are observed with primetarget delays of milliseconds and seconds (Kristjánsson and Campana, 2010), days (Summerfield et al., 2006), and even months (DeSchepper and Treisman, 1996).

The search display contained two different colored circles above and below central fixation, each containing a gap $\left(0.865^{\circ}\right.$ of visual angle from center to center of each circle). The task was to find the circle containing a horizontal gap and identify its position (left or right side of the circle); the circle with a gap in the vertical object plane was a distracter. In valid trials, the target circle matched the color of the memory cue, whereas in invalid trials the memory cue matched the color of the distracter. The circles were drawn in five different colors (red, blue, pink, green, or yellow) with a diameter of $0.541^{\circ}$ of visual angle and presented at the center of the screen. The search display was small (the total area covered by the two circles was $1.4^{\circ}$ ) to be projected in the fovea, and its duration was titrated for each participant during the training phase to obtain an accuracy level of $80-90 \%$ (duration ranged from 26.67 to $66.67 \mathrm{~ms}$ ). Each circle was masked by a $106 \mathrm{~ms}$ array composed of four squares of different colors, randomly selected with the constrain that the memory color never appeared as part of the mask. In the WM condition, a color memory test followed the search task. A colored circle appeared, and participants responded "same" or "different" relative to the memory cue. Search responses preceded memory test responses and had a deadline of $1500 \mathrm{~ms}$ from search onset. Memory test display was presented until response.
Two TMS conditions were included: visual cortex and sham TMS. There were 36 trials for each combination of TMS, cue validity, and memory requirement conditions. The experiment was performed in four blocks of 36 trials (each block contained 18 valid and 18 invalid trials). The order of blocks was counterbalanced between subjects; half of the participants began with a sham TMS block, and the other half began with early visual cortex (EVC) TMS. Half of the participants performed the WM blocks first, and the other half performed the priming blocks first.

TMS and site localization. Biphasic TMS pulses were delivered by using a MagPro R 30 stimulator (Medtronic Functional Diagnostics A/S; maximum stimulator output, 1.9 T) and a figure-eight TMS coil (MC-B70; MagVenture). The coil was fixed on a custom-made holder placed tangentially on the skull. The orientation of the handle was horizontal, pointing toward the occipital lobe. The coil was placed 2 $\mathrm{cm}$ above the inion. This location closely matches the brain areas V1 and V2 (Salminen-Vaparanta et al., 2011). We thus refer to the stimulation site as the EVC.

TMS-induced phosphenes were reported by participants to occur in the center of their visual field; thus the application TMS likely affected the area of the occipital cortex associated with the visual field of the critical stimulus (Pascual-Leone and Walsh, 2001) because the search array was presented in the fovea. We further confirmed that our TMS procedure targeted the EVC by means of neuronavigation performed in two of the participants in the experiment and a new observer that did not take part in the study. We used the BrainVoyager TMS Neuronavigator (Brain Innovation) that uses stereotaxic data based on an ultrasound-based coregistration system. We obtained structural MRI scans by using a Siemens Magnetom Verio 3T MRI scanner; 176 T1 weighted anatomical sagittal images were acquired with an FOV of $220 \times 220 \mathrm{~mm}$, TR of 1900 $\mathrm{ms}$, TE of $2.48 \mathrm{~ms}$, and slice thickness of $1 \mathrm{~mm}$, leading to a voxel resolution of $1 \times 1 \times 1 \mathrm{~mm}$. Figure 2 illustrates the occipital positions in the three participants.

Phosphene thresholds were identified individually by using a modified binary search algorithm (Tyrrell and Owens, 1988) that gives a TMS intensity value at which phosphenes are reported on $50 \%$ of trials. Mean intensity for inducing phosphenes was $45 \%$ of the maximum stimulator output. In the main experiment, subjects were stimulated at $90 \%$ of phosphene threshold. No participant reported phosphenes during the experiment.

For sham TMS, the coil was placed perpendicularly over the stimulation site used in the EVC-TMS condition such that one wing of the coil 


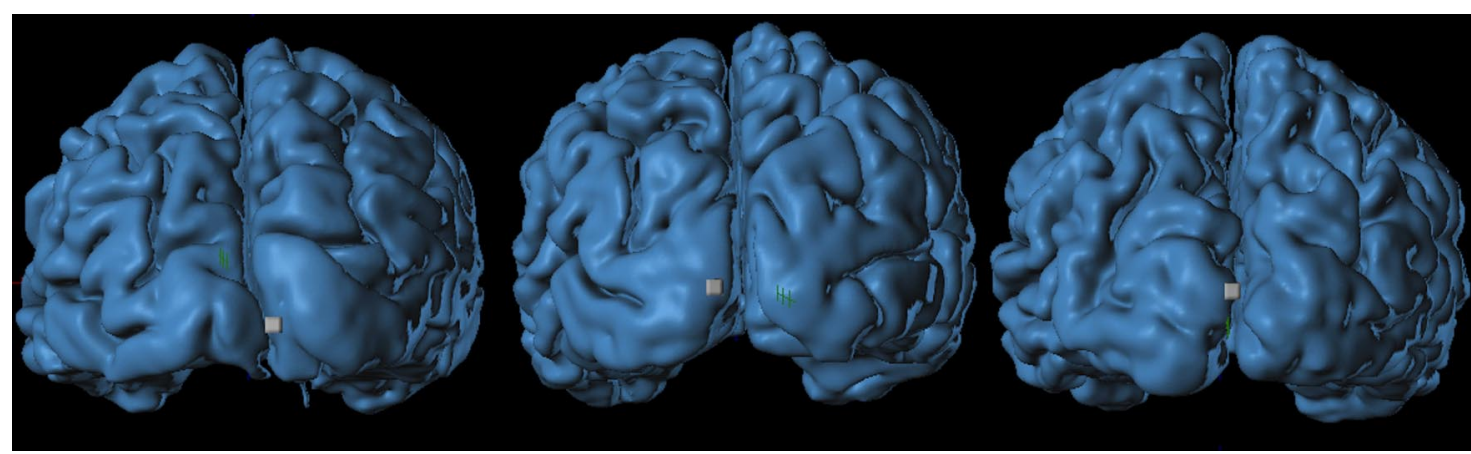

Figure 2. Illustration of occipital target sites.

made contact with the scalp. This condition controlled for the auditory artifacts induced by TMS. Three pulses were applied at $10 \mathrm{~Hz}$ with the onset of the search display.

\section{Results}

\section{WM accuracy}

Mean accuracy was $91 \%$ correct. There were no main effects of TMS and validity $\left(F_{(1,11)}=0.025, p=0.8\right.$ and $F_{(1,11)}=2.17, p=$ $0.17)$ and no interaction between factors $\left(F_{(1,11)}=0.2, p=0.6\right)$.

\section{Search reaction times}

Only correct responses in both search and memory tasks were included in these analyses. In the priming condition participants withheld responses to the task on $87 \%$ of catch trials. A 2 (memory requirement: $\mathrm{WM}$, priming) $\times 2$ (cue validity: valid, invalid) ANOVA on median RTs in the sham condition was conducted to confirm the behavioral pattern typically found in this paradigm. There were no main effects of memory condition $\left(F_{(1,11)}<1\right)$ or validity $\left(F_{(1,11)}=2.29, p=0.15\right)$ but a significant interaction between factors $\left(F_{(1,11)}=6.58, p=0.026\right)$; cue validity effects on RTs were higher in the WM relative to the priming condition (Fig. 3A). Paired $t$ tests showed faster RTs on valid relative to invalid trials in the WM case $\left(t_{(11)}=3.15, p=0.009\right)$ but not in the priming condition $\left(t_{(11)}=1.12, p=0.28\right)$. WM contents biased attention (Soto et al., 2007).

Next, we performed a 2 (memory requirement) $\times 2$ (cue validity) $\times 2$ (TMS: EVC or sham) ANOVA. There was a main effect of the memory requirement $\left(F_{(1,11)}=6.01, p=0.026\right)$ with search being overall slower in the WM relative to the priming condition. There was no main effect of TMS $\left(F_{(1,11)}=1.04, p=\right.$ $0.33)$ and no main effect of validity $\left(F_{(1,11)}=0.37, p=0.56\right)$. There was an interaction between the memory requirement and TMS $\left(F_{(1,11)}=5.79, p=0.035\right)$, indicating that overall disruption of search latencies by TMS was higher in the WM case $(p=0.045)$ than in the priming case $(p=0.244)$. The remaining interactions were not significant ( $p=0.39$ ). In summary, TMS did not modulate the cue-validity effect on RTs.

\section{Search accuracy}

We performed a 2 (memory requirement) $\times 2$ (TMS) $\times 2$ (validity) ANOVA on the proportion of correct search responses. In the WM case, we included only trials with correct responses in the memory test. There was no effect of memory condition $\left(F_{(1,11)}=0.01, p=0.9\right)$, TMS $\left(F_{(1,11)}=0.26, p=\right.$ $0.6)$, or validity $\left(F_{(1,11)}=0.14, p=0.7\right)$. There was an interaction between memory requirement and validity $\left(F_{(1,11)}=\right.$ $7.83, p=0.017)$ and a three-way interaction between all factors $\left(F_{(1,11)}=7.94, p=0.017\right)$. As can be seen in Figure $3 B$,
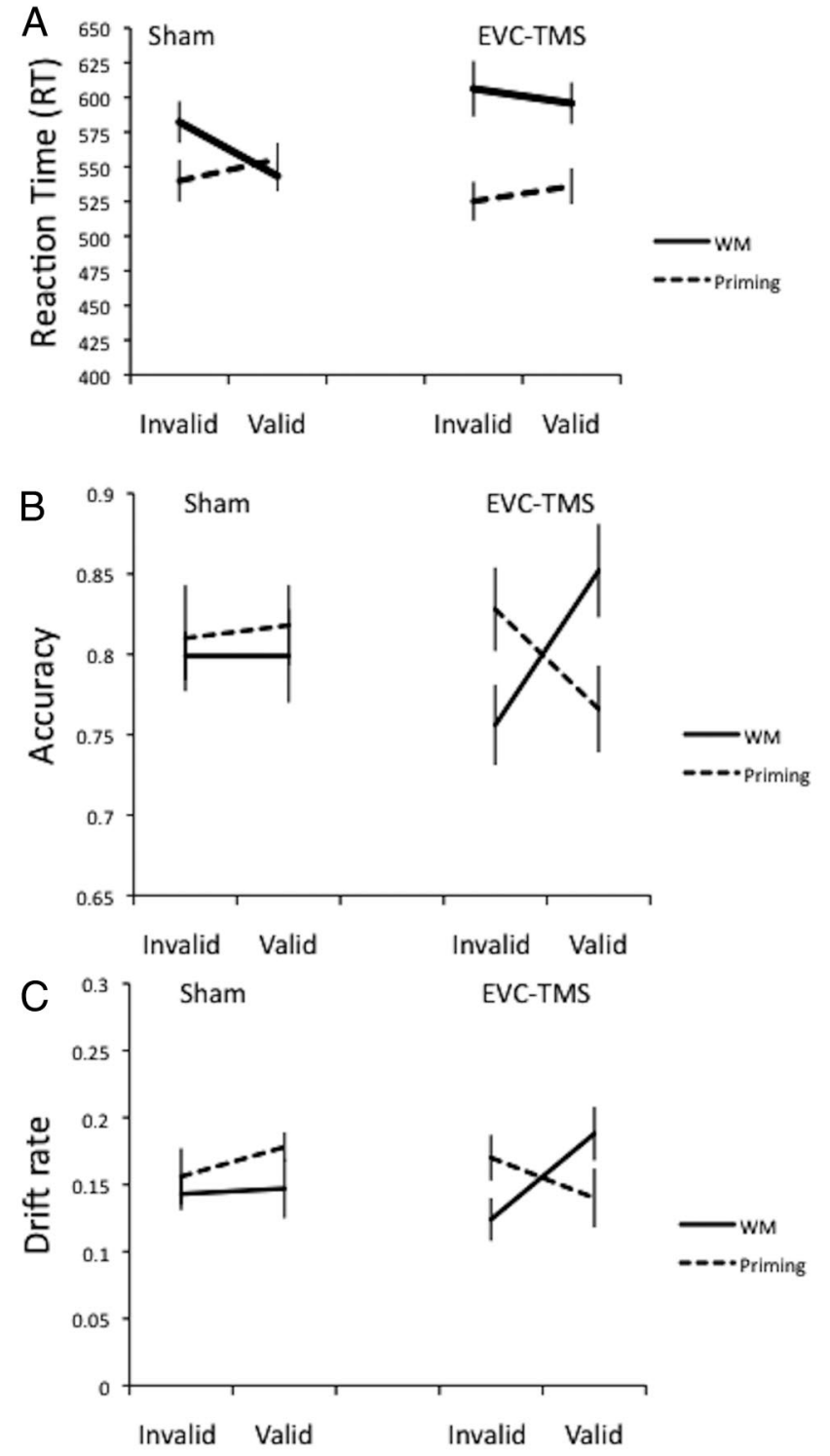

Figure 3. A, Search RTs as a function of TMS site, memory requirement, and validity. Error bars reflect SE from which between-subjects variance has been removed (Loftus and Masson, 1994). B, Mean proportion correct. C, Drift rate.

EVC TMS modulated the effect of cue validity in opposite ways depending on the memory requirement. When occipital TMS was applied in the WM condition search accuracy was facilitated on valid relative to invalid trials $\left(t_{(11)}=2.621, p=\right.$ 
0.024); in the priming condition occipital TMS tended to facilitate search accuracy in invalid rather than valid trials $\left(t_{(11)}=1.951, p=0.074\right)$, and this trend was more significant when the overall cueing effect in the priming condition (i.e., accuracy invalid - accuracy valid) was compared between occipital TMS and sham conditions $\left(t_{(11)}=2.36, p=0.038\right)$. Interestingly, there were no validity effects on search accuracy in the sham condition. Validity effects emerged with the application of occipital TMS.

Occipital TMS elevated the RTs in the WM case relative to the priming case; one may question whether the TMS modulation of the validity effects in search accuracy may arise from a speedaccuracy tradeoff. We first note that although TMS led to slower RTs in the WM condition, there was no interaction between TMS and validity on RTs. That TMS modulated the validity effects on search accuracy and that this was a function of the memory requirement (WM vs priming) may reflect on the distinct effects of TMS on RTs and accuracy. The interaction between TMS and validity appears more specific to search accuracy. Although validity effects on RTs were higher in the WM relative to the priming condition, paired $t$ tests showed that WM valid-invalid RT differences were significant in the sham TMS $\left(t_{(11)}=3.15, p=\right.$ $0.009)$ but not in the WM-TMS case $\left(t_{(11)}=0.42, p=0.683\right)$; in the latter case the TMS modulation of the validity effect was evident only in accuracy. Taken together, the pattern of results indicates that the TMS effect do not reflect speed-accuracy tradeoff.

\section{Diffusion model analysis}

We used a diffusion model approach (Wagenmakers et al., 2007) to derive information about (1) the observer's sensitivity to the relevant stimulus (the "drift rate" parameter), (2) the decision threshold ("boundary" parameter, which here would reflect the amount of information needed to trigger a left or right response), and (3) mean of "nondecision" time, which refers to the duration of information processing before the decision process and the time taken to execute the motor command. These analyses allowed us to learn whether the pattern of results were linked to sensitivity to the memory-matching item (drift rate) but critically incorporating information from both RT and accuracy. This analysis is particularly relevant here because the effects of TMS were not reflected similarly on RT and accuracy measures. This approach has been used in a previous TMS study (Cohen Kadosh et al., 2010).

The analysis of the drift rate confirmed the pattern found in the accuracy data, with a significant interaction between memory requirement, validity, and TMS $\left(F_{(1,11)}=21.02, p=0.001\right)$. None of the other interactions or main effects approached significance (lowest $p$ value $=0.24$ ). To understand the source of the three-way interaction we computed the validity effects on drift rate across the different conditions and then assessed the effects of TMS as a function of memory state by means of paired $t$ tests. In the WM condition, occipital TMS increased the validity effect relative to sham $\left(t_{(11)}=2.56, p=0.027\right)$. The opposite pattern was observed in the priming condition, with occipital TMS inducing a negative validity effect relative to sham TMS $\left(t_{(11)}=\right.$ $-2.9, p=0.011$; Fig. $3 C$ ). Importantly, these results indicate that occipital TMS influenced the sensitivity to memory matches rather than reflecting changes in decisional criteria, strategies to solve the task, or any speed-accuracy tradeoff. The analysis of boundary separation (i.e., decision criteria) revealed a main effect of the memory requirement $\left(F_{(1,11)}=5.61, p=0.037\right)$; this parameter of the model was higher in the WM than in the priming condition, suggesting that less evidence about the target was required in the priming condition before decision making. Importantly, there were no further trends for other main effects or interactions between memory requirement, validity, or TMS (lowest $p$ value $=0.21$ ). Thus neither validity nor TMS influenced decision biases. No significant main effects or interaction effects were found on the nondecision time (lowest $p$ value $=$ $0.174)$.

\section{Discussion}

Nonpredictive color cues in WM guided target selection, as indexed by faster search latencies in valid relative to invalid trials. In contrast, mere repetition priming had no effect on selection (Olivers et al., 2006; Soto et al., 2011). The absence of priming effects here contrasts with the literature on priming in visual search (Kristjánsson and Campana, 2010) in which target selection on a previous trial modulates performance in subsequent trials for targets carrying similar features. A fundamental difference between previous priming studies and our study is that here the prime was not correlated with the sought target. Under these circumstances, attention may be directed away from the primed representation during search, thus explaining the absence of priming effects. In studies of intertrial priming, the primed features may not be inhibited because they often match the target of selection. Note also that our requirement to attend the cue should have been sufficient to trigger priming because this can occur without conscious awareness of the prime (Kristjánsson et al., 2005).

The main finding was that occipital TMS modulated the impact of memory cueing on selection in opposite directions depending on memory state. The influence of TMS reflected modulation at a perceptual level, enhancing and impairing sensitivity to a memory-matching item depending on the memory context. The TMS effect was not reflected in RTs or other measures of decisional criteria. In the priming condition, TMS led to reduced search accuracy on valid relative to invalid trials. This finding suggests that occipital TMS impaired the representation of the primed item during search; this would interfere with selection on valid trials, but it would facilitate selection on invalid trials because here the impairment of a distracter representation ought to facilitate target selection. Conversely, in the WM condition TMS enhanced search accuracy on valid relative to the invalid trials, which is consistent with occipital TMS boosting the capture of attention by WM-matching items.

These distinct TMS effects on selection may be associated with the neural state of the visual cortex as a function of memory context. According to this view, occipital TMS may boost attention toward WM-matching items because these representations are enhanced in visual cortex. This is supported by evidence that TMS can strengthen the representation of items held in visual short-term memory. Specifically, the application of TMS over the visual cortex has been shown to selectively enhance the detection of features that participants are actively holding in memory (Cattaneo et al., 2009; Silvanto and Cattaneo, 2010); instead, in classic priming paradigms TMS can impair the detection of merely primed features (Campana et al., 2002, 2006).

As noted in the introduction, previous work did not address whether visual cortical engagement is associated with the maintenance of a visual memory trace or whether it can also play a role in attentional biasing. This is because most of these prior studies used cues that specified the target of selection, 
thus conflating memory and attention responses (for discussion, see Soto et al., 2007). The same concern applies to single unit evidence that WM and priming are associated with enhancement and attenuation of neuronal activity in inferior temporal and visual cortices through the reappearance of a memorymatching item (Brown and Xiang, 1998). We propose that WMassociated enhancement of neuronal activity may render the neurons representing the WM content more susceptible to TMS relative to other neurons in the targeted area. Selective activation of neurons engaged in WM would increase the signal-to-noise ratio of the memory representation and would facilitate attentional capture by features matching the WM contents. This would not hold in the priming condition that is likely associated with attenuation of neuronal activity in visual cortex with repetition. Different models have been proposed to account for repetition attenuation effects, including the "sharpening" and "fatigue" models (Grill-Spector et al., 2006). Repetition attenuation in visual cortex is very specific to the properties of the visual representation and has not been associated with high-level priming and behavioral response facilitation (Schacter et al., 2007). Notably, neural repetition attenuation effects in visual regions appear more consistent with sharpening of the neural response (Wiggs and Martin, 1998), whereby responses to repeated items are sharper, whereas responses for nonrepeated items are "suppressed." Under this conditions, TMS may decrease the signal-to-noise ratio of the primed item because, for example, it may activate the suppressed neurons other than the primed ones (Silvanto et al., 2008). Then, a selective impairment in the processing of the primed features with TMS would result (Campana et al., 2002, 2006). Further research using concurrent fMRI and online TMS protocols is needed to elucidate how TMS modulates neural activity in the presence of stimulus repeats and how this varies as a function of current mnemonic demands.

In contrast to the effects of TMS on search, there were no TMS effects on memory indicating that occipital TMS did not influence search performance by modulating the memory trace in visual regions; were this the case, a similar level of performance should have been observed in valid and invalid trials. Occipital TMS therefore modulated the impact of memory biasing signals in the early visual cortex, thus driving selection. We also note that, although validity effects on search accuracy were not evident in sham TMS conditions, our evidence demonstrates that occipital TMS nevertheless interacted with the memory task requirement (WM vs priming) to modulate attentional biases. A similar dissociation induced by TMS has been found previously. For example, studies using visual adaptation showed that behavioral effect of adaptation was not evident in the control condition, yet an interaction between adapter type and TMS effects was found (Cattaneo et al., 2008, 2010; Cohen Kadosh et al., 2010). Adaptation, priming, or WM may alter neural activation states in visual regions, but the functional relevance of these neural states may be revealed only by the interaction with TMS.

The visual cortex is one of the neural regions identified in recent functional MRI studies as part of a network involved in memory guidance, including temporal, superior frontal (Soto et al., 2007, 2012; Grecucci et al., 2010), ventral inferior frontal gyrus (Soto et al., 2011), and the thalamus (Rotshtein et al., 2011). Occipital TMS effects may not be local, because TMS may spread across extra-striate regions and lead to distal activity changes in neural regions associated with attention. In any case, this would not undermine the relevance of our novel observation, namely, the pivotal role of early visual cortical engagement in featurebased attentional biasing through memory.

\section{References}

Brown MW, Xiang JZ (1998) Recognition memory: neuronal substrates of the judgement of prior occurrence. Prog Neurobiol 55:149-189.

Campana G, Cowey A, Walsh V (2002) Priming of motion direction and area V5/MT: a test of perceptual memory. Cereb Cortex 12:663-669.

Campana G, Cowey A, Walsh V (2006) Visual area V5/MT remembers "what" but not "where". Cereb Cortex 16:1766-1770.

Cattaneo L, Sandrini M, Schwarzbach J (2010) State-dependent TMS reveals a hierarchical representation of observed acts in the temporal, parietal, and premotor cortices. Cereb Cortex 20:2252-2258.

Cattaneo Z, Rota F, Vecchi T, Silvanto J (2008) Using state-dependency of transcranial magnetic stimulation (TMS) to investigate letter selectivity in the left posterior parietal cortex: a comparison of TMS-priming and TMS-adaptation paradigms. Eur J Neurosci 28:1924-1929.

Cattaneo Z, Vecchi T, Pascual-Leone A, Silvanto J (2009) Contrasting early visual cortical activation states causally involved in visual imagery and short-term memory. Eur J Neurosci 30:1393-1400.

Chawla D, Rees G, Friston KJ (1999) The physiological basis of attentional modulation in extrastriate visual areas. Nat Neurosci 2:671-676.

Cohen Kadosh R, Muggleton N, Silvanto J, Walsh V (2010) Double dissociation of format-dependent and number-specific neurons in human parietal cortex. Cereb Cortex 20:2166-2171.

DeSchepper B, Treisman A (1996) Visual memory for novel shapes: implicit coding without attention. J Exp Psychol Learn Mem Cogn 22:27-47.

Desimone R, Duncan J (1995) Neural mechanisms of selective visual attention. Annu Rev Neurosci 18:193-222.

Grecucci A, Soto D, Rumiati RI, Humphreys GW, Rotshtein P (2010) The interrelations between verbal working memory and visual selection of emotional faces. J Cogn Neurosci 22:1189-1200.

Grill-Spector K, Henson R, Martin A (2006) Repetition and the brain: neural models of stimulus-specific effects. Trends Cogn Sci 10:14-23.

Harrison SA, Tong F (2009) Decoding reveals the contents of visual working memory in early visual areas. Nature 458:632-635.

Kristjánsson A, Campana G (2010) Where perception meets memory: a review of repetition priming in visual search tasks. Atten Percept Psychophys 72:5-18.

Kristjánsson A, Vuilleumier P, Malhotra P, Husain M, Driver J (2005) Priming of color and position during visual search in unilateral spatial neglect. J Cogn Neurosci 17:859-873.

Loftus GR, Masson MEJ (1994) Using confidence intervals in withinsubject designs. Psychon Bull Rev 1:476-490.

Martinez-Trujillo JC, Treue S (2004) Feature-based attention increases the selectivity of population responses in primate visual cortex. Curr Biol 14:744-751.

Miller EK, Desimone R (1994) Parallel neuronal mechanisms for shortterm memory. Science 263:520-522.

Motter BC (1994) Neural correlates of attentive selection for color or luminance in extrastriate area V4. J Neurosci 14:2178-2189.

Olivers CN, Meijer F, Theeuwes J (2006) Feature-based memory-driven attentional capture: visual working memory content affects visual attention. J Exp Psychol Hum Percept Perform 32:1243-1265.

Pascual-Leone A, Walsh V (2001) Fast backprojections from the motion to the primary visual area necessary for visual awareness. Science 292:510-512.

Pasternak T, Greenlee MW (2005) Working memory in primate sensory systems. Nat Rev Neurosci 6:97-107.

Rotshtein P, Soto D, Grecucci A, Geng JJ, Humphreys GW (2011) The role of the pulvinar in resolving competition between memory and visual selection: a functional connectivity study. Neuropsychologia 49:1544-1552.

Salminen-Vaparanta N, Noreika V, Revonsuo A, Koivisto M, Vanni S (2011) Is selective primary visual cortex stimulation achievable with TMS? Hum Brain Mapp. Advance online publication. Retrieved February 5, 2012. doi:10.1002/hbm.21237.

Schacter DL, Wig GS, Stevens WD (2007) Reductions in cortical activity during priming. Curr Opin Neurobiol 17:171-176.

Silvanto J, Cattaneo Z (2010) Transcranial magnetic stimulation reveals the 
content of visual short-term memory in the visual cortex. Neuroimage 50:1683-1689.

Silvanto J, Muggleton N, Walsh V (2008) State-dependency in brain stimulation studies of perception and cognition. Trends Cogn Sci 12:447-454.

Soto D, Humphreys GW, Rotshtein P (2007) Dissociating the neural mechanisms of memory-based guidance of visual selection. Proc Natl Acad Sci U S A 104:17186-17191.

Soto D, Greene CM, Chaudhary A, Rotshtein P (2011) Competition in working memory reduces frontal guidance of visual selection. Cereb Cortex. Advance online publication. Retrieved February 5, 2012. doi:10.1093/cercor/bhr190.

Soto D, Rotshtein P, Hodsoll J, Mevorach C, Humphreys GW (2012) Com- mon and distinct neural regions for the guidance of selection by visuoverbal information held in memory: Converging evidence from fMRI and rTMS. Hum Brain Mapp 33:105-120.

Summerfield JJ, Lepsien J, Gitelman DR, Mesulam MM, Nobre AC (2006) Orienting attention based on long-term memory experience. Neuron 49:905-916.

Tyrrell RA, Owens DA (1988) A rapid technique to assess the resting states of eyes and other threshold phenomena: the Modified Binary Search (MOBS). Behav Res Methods 20:137-141.

Wagenmakers EJ, van der Maas HL, Grasman RP (2007) An EZ-diffusion model for response time and accuracy. Psychon Bull Rev 14:3-22.

Wiggs CL, Martin A (1998) Properties and mechanisms of perceptual priming. Curr Opin Neurobiol 8:227-233. 